\section{A marriage is consummated}

\section{Peter A. Lawrence and Andrew Tomlinson}

PICTURE a child's jigsaw puzzle - the kind with a few large pieces, but with a significant bit missing. The jigsaw analogy is apt for the insect compound eye, where not only do all the cells fit together intricately, but the pieces of understanding interlock in such a satisfying way that they leave precisely shaped holes that ask to be occupied. One such space is now filled by Krämer et al. (see page 207 of this issue ${ }^{1}$ ), who have apparently found the ligand for the sevenless protein.

The Drosophila eye consists of hundreds of repeating units called ommatidia, and each one is made as a unit ${ }^{2}$. Initially, one set of photoreceptor cells called R8 are selected from a sheet of epidermis $^{2}$. This process probably depends on lateral inhibition ${ }^{3}$ (the mechanism by which individual cells are selected from a population and, once selected, develop in a new direction and also inhibit nearby cells from doing the same $)^{4}$. After an R8 cell is formed, the ommatidium builds outwards as adjoining epidermal cells are recruited in a stereotyped pattern, first R2 and R5, then R3, R4 and R1, R6, and finally R7 (refs 2,5). The other cells of the ommatidium, the cone cells that form the lens, the pigment cells that insulate each ommatidium from its neighbours and the founding cells of eye bristles, are all added on by further recruitment. When this sequence is complete, there are still a group of surplus unallocated cells; these die to leave the whole structure as perfect and as precise as a crystal ${ }^{6}$.

Because the eye is dispensable, mutations which damage it will not be lethal to the fly. So genes that effect specific steps in this process have been identified. For example, the rough gene is needed to specify cells R2 and R5. In its absence they do not develop properly and cannot trigger the events that would normally occur next ${ }^{7}$.

The recruitment of the $\mathrm{R} 7$ cell has been especially well investigated, beginning with the isolation of sevenless ${ }^{-}$flies in 1976 (ref.8); these mutants have no R7 cells and the cell that would have made the $\mathrm{R} 7$ cell makes a cone cell instead ${ }^{9}$. Studies with genetic mosaics show that the sevenless gene is needed only in the R7 cell itself, so the requirement for the gene is cell autonomous ${ }^{8}$. The sevenless gene encodes a tyrosinekinase receptor protein that is predicted to be in the membrane ${ }^{10}$. In the electron microscope, the sevenless antigen can be seen on the apical plasma membrane of the R7 cells, and seems to form patches where the R7 cell touches the founding
R8 cells ${ }^{11}$. This is a striking visual image, one which suggests that there is a ligand on the R8 cell that binds to the sevenless protein molecules on the R7 cell and thereby creates and tethers rafts of re-

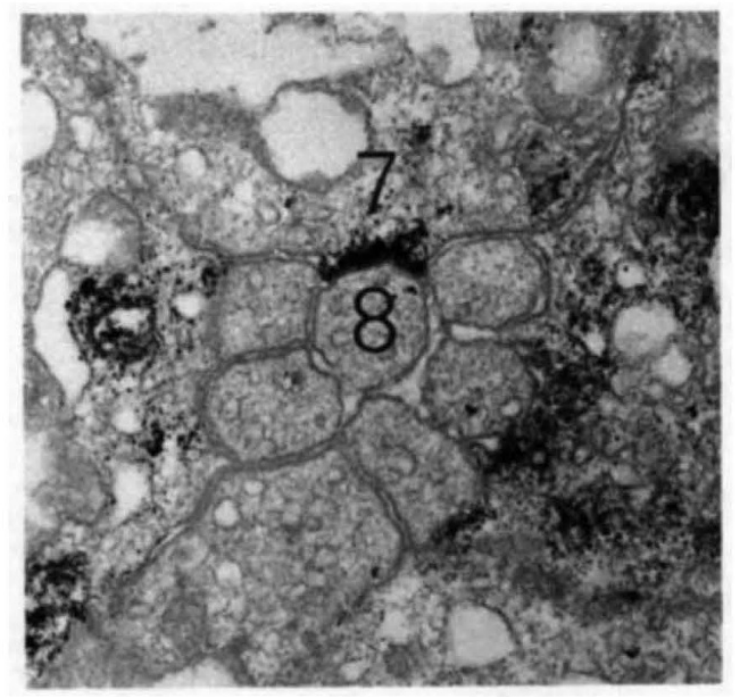

Developing photoreceptor cells in the Drosophila eye; sevenless antigen is seen where R7 contacts R8. ceptor protein (see figure).

Three years ago, Zipursky's group identified a gene that might encode this ligand and called it bride of sevenless (boss $)^{12}$. Mutations in boss cause a phenotype identical to that caused by mutations in sevenless, but analysis of eyes that are a mixture of boss ${ }^{+}$and boss cells show that the gene is not needed in the R7 cell itself. The requirement for boss is therefore not cell autonomous. Instead, the phenotype of each individual ommatidium correlates with the genotype of the R8 cell. If the R8 cell is boss $^{+}$, the ommatidium will have the full complement of eight photoreceptor cells, if it is boss ${ }^{-}$it will have seven, and these rules apply whatever the genotypes of the other photoreceptor cells. The genetic mosaics suggest that the boss gene product should be looked for in the R8 cells ${ }^{12}$

The boss gene encodes an integral membrane protein with seven transmembrane domains $^{13}$, and Kramer et al. ${ }^{1}$ now offer persuasive arguments that this protein is indeed the ligand for sevenless. First, using electron microscopy they show that the protein is located on the apical surfaces of the R8 cells. It is also found inside the R8 cells in smaller multivesicular bodies and, significantly, inside each $\mathrm{R} 7$ cell in an extraordinarily large membrane-bound body - so big it is easily seen in the light microscope. In the absence of the sevenless gene, the boss antigen is not found in the R7 cells.
Second, they show that the boss and sevenless proteins can interact; when they are expressed separately in transfected tissue culture cell lines the cells do not stick together, but when the two cell lines are mixed they form aggregates.

In Drosophila molecular genetics, it is commonplace for the ligand to be known but not the receptor (or vice versa). Here is a welcome exception. Moreover this is an example of molecular transduction not between a diffusing ligand and a membrane-bound receptor, but, apparently, between two integral membrane proteins. If it is, how does the boss antigen enter the R7 cell? Further questions requiring answers are, for example, why does only the prospective R7 cell take up the boss protein - other cells that express the sevenless protein are in contact with R8, and yet do not appear to contain the antigen. Krämer et al. say that the uptake of the boss protein might be an important part of the signalling process. If this is so it cannot be essential, as cells which contain artificially high amounts of sevenless protein can be self-activated and transform into R7 cells ${ }^{14}$ - moreover this self-activation can occur in the absence of the boss gene ${ }^{14}$. So, how does the boss protein actually produce the change in fate of the cell? And what is the huge 'multivesicular body' in the R7 cell?

The work discussed here is the latest in a line of imaginative and illuminating studies carried out by Zipursky's group. They illustrate how, increasingly, genetic analysis of pattern formation takes us from the relatively clear logic of genetics into the thickets of cell biology.

Peter A. Lawrence and Andrew Tomlinson are in the MRC Laboratory of Molecular Biology, Hills Road, Cambridge CB2 2QH, UK.

1. Krämer, H., Cagan, R.L. \& Zipursky, S.L. Nature 352 207-212 (1991).

2. Tomlinson, A. \& Ready, D.F. Dev/ Biol. 120, 366-376 (1987).

3. Cagan, R.L. \& Ready, D.F. Genes Dev. 3, 1099-1112 (1989).

4. Simpson, P. Development 109, 509-519 (1990)

5. Tomlinson, A. Development 104, 183-193 (1988).

6. Cagan, R.L. \& Ready, D.F. Devi Biol. 136, 346-362 (1989).

7omlinson, A., Kimmel, B.E. \& Rubin, G.M. Cell 55 771-784 (1988).

8. Harris, W.A., Stark, W.S. \& Walker, J.A. J. Physiol. 256, 415-439 (1976).

9. Tomlinson, A. \& Ready, D.F. Science 231, 400-402 (1986)

10. Hafen, E., Basler, K., Edstroem, J.-E. \& Rubin, G.M. Science 236, 55-63 (1987).

11. Tomlinson, A., Bowtell, D.D.L., Hafen, E. \& Rubin, G Cell 51, 143-150 (1987).

12. Reinke, R. \& Zipursky, S. L. Cell 55, 321-330 (1988)

13. Hart, A.C., Krämer, H., Van Vactor, D.L.,Jr, Paidhungat, M. \& Zipursky, S.L. Genes Dev. 4, 1835-1847 (1990).

14. Basler, K., Christen, B. \& Hafen, E. Cell 64, 1069 1081 (1991). 